\title{
Diabetes mellitus and female urinary incontinence: a time for change
}

\author{
Mohamed Abdel-fattah • Diaa E. E. Rizk
}

Received: 2 March 2012 / Accepted: 4 April 2012 /Published online: 15 May 2012

(C) The International Urogynecological Association 2012

Diabetes mellitus (DM), a chronic metabolic disease, is becoming a global health problem with increasing prevalence. The incidence of type I DM is fairly constant but that of type II $\mathrm{DM}$ is on the rise, most likely as a consequence of the contemporary changes in lifestyle, increasing obesity rates, and aging of the female population. DM is considered an independent risk factor for female urinary incontinence (UI); in a pooled analysis of 71,650 women aged 37-79 years, Danforth et al. [1] showed a $20 \%$ increased odds of UI in women with DM [odds ratio (OR) 1.2, $95 \%$ confidence interval (CI) 1.0 $1.3, p=0.01)]$ This was largely because of urgency UI with no apparent association with stress or mixed UI. In a study of the Turkish population, Izci et al. [2] has shown a 2.5-fold increase in prevalence of UI in diabetic women, mainly of mixed UI. Similar results were shown in studies from Taiwanese, Chinese, and South American female populations. Despite this evidence, the management of incontinent diabetic women has been relatively neglected by the urogynecology community. This is partly because the etiology of DM is not well understood and partly due to lack of training in diabetic medicine in most urogynecology training programs.

\footnotetext{
M. Abdel-fattah

Division of Applied Health Sciences, University of Aberdeen, Aberdeen, UK

D. E. E. Rizk

Department of Obstetrics and Gynecology, Ain Shams University, Cairo, Egypt

D. E. E. Rizk

The Canadian Continence Foundation, Peterborough, ON, Canada

M. Abdel-fattah $(\bowtie)$

Second Floor, Aberdeen Maternity Hospital,

Foresterhill, Aberdeen AB25 2ZD, UK

e-mail: m.abdelfattah@abdn.ac.uk
}

Traditionally, diabetic bladder dysfunction has been described as a triad of decreased sensation, increased capacity, and poor emptying causing a broad spectrum of symptoms including overactive bladder $(\mathrm{OAB})$, voiding dysfunction, and urinary retention [3]. The role of DM in the pathophysiology of OAB and urgency UI is well described as a result of glycosuria, recurrent urinary tract infections (UTI), diabetic autonomic neuropathy, and diabetic cystopathy [4-6]. Hyperglycemia will often cause glycosuria and consequently osmotic diuresis leading to polyuria and increased frequency of micturition in diabetic women [7]. Autonomic neuropathy is a late complication of DM with a cumulative increase in prevalence over time [8]. Liu et al. [9] have recently shown that the prevalence of lower urinary tract symptoms is directly related to duration of DM with 2.4- and 4.2-fold increase with DM duration $>10$ years and age $>50$ years, respectively. The cause of diabetic neuropathy is multifocal and includes ischemia, superoxide-induced free radical formation, impaired axonal transport, and metabolic derangement of the Schwann cell resulting in segmental demyelination and impairment of nerve conduction [9]. Animal and research data suggest that diabetic cystopathy is caused by ultrastructural and microvascular damage to the detrusor muscle with alteration in its neural component ultimately resulting in alterations in detrusor muscle function [10]. Diabetic cystopathy affects up to $40-80 \%$ of diabetic patients, is slowly progressive, and clinically characterized by decreased bladder sensation and either impaired or unstable detrusor contractions [8]. The metabolic syndrome associated with DM and characterized by dyslipidemia and hypertension can further decrease the blood flow to the detrusor muscle and compromise bladder function. Liu et al. [9] have shown that patients with DM and metabolic syndrome showed a higher trend for developing urgency UI. These studies provide an explanation for the fact that $\mathrm{OAB}$ is the main clinical feature of diabetic cystopathy which can also be associated with increased post-void residual 
urine volumes (PVR), decreased bladder sensation, and reduced detrusor contractility due to damage to afferent nerve fibers ultimately leading to detrusor hypocontractility and urinary retention [10].

DM does not seem to directly contribute to the development of stress UI. However, autonomic neuropathy affecting the innervations of the striated muscle of the urethral sphincter and pelvic floor muscles can lead to the development of an incompetent urethral mechanism and consequently stress UI [11]. Obesity, which is often associated with type $2 \mathrm{DM}$, is also a well-known risk factor for development of stress UI due to increased intra-abdominal and pelvic pressure [12]. Causes of stress UI in diabetic women also include the effects of gestational diabetes and delivery conditions such as macrosomia, prolonged second stage of labor, and the increased risk of instrumental delivery on the pelvic floor. Both obesity and DM have been shown, in a number of studies, to be associated with increased risk of failure for surgical treatment using midurethral slings [11]. Weight loss, in obese patients, through diet and exercise, has long been advocated as an integral part of the conservative management plan for women with UI. Interestingly, a number of studies have also shown that the development of DM can be prevented by lifestyle interventions like exercise and diet regulation with a consequent decrease in the overall prevalence of female UI by nearly $50 \%[11,12]$.

Management of women with DM and UI involves a proper workup with history, examination, and laboratory investigations. Urinalysis and culture to rule out bacterial cystitis and biochemical tests to assess diabetic control and end organ damage should be performed. Although the latter is good clinical practice, tight glycemic control was not shown to be associated with less bothersome OAB [9]. A complete urogynecological examination should be followed by urodynamic studies if invasive treatment is contemplated. Management strategies should include lifestyle interventions like weight loss from dietary modification and exercise, cessation of smoking, and optimization of volume and type of fluids taken. Data analysis from the Diabetes Prevention Program, a large randomized controlled trial from 27 centers in the USA [12], showed that obese women at risk of DM and UI in the active lifestyle intervention group benefitted from dietary modification and exercise and reduced their odds of suffering from UI as compared to women in the metformin or placebo group. Pelvic floor exercise and bladder retraining have been shown to be effective first-line management in women with UI in general and would seem to be appropriate in diabetic women. There is no robust evidence, however, for the effectiveness of both treatment modalities, or lack of it, in this patient subgroup. Women who suffer from reduced bladder sensation are advised to carry out timed voiding on a regular basis. Intermittent selfcatheterization with or without antibiotic cover may be required in cases of high PVR and recurrent UTI. Pharmacological treatment options such as anticholinergic drugs have been proven to be effective in the management of women with $\mathrm{OAB}$ with no evidence to suggest otherwise in diabetic women.

In conclusion, the findings of clinical and basic science studies emphasize the important link between UI and DM in women and the public health responsibility of urogynecologists to actively participate in the management of DM. Therefore, it is time for the urogynecology arena to appreciate the importance of DM in the specialty and start thinking about the need to change the current practice. In particular, all diabetic women seeking health care advice should be given information about UI and possible strategies for its prevention and management. Similarly, incontinent women with risk factors for DM such as obesity and family history can be offered screening for DM. Urogynecologists should participate in health promotion strategies for DM such as modification of lifestyle, weight reduction, and exercise in all their patients and ensure that their diabetic patients have good diabetic control.

\section{References}

1. Danforth KN, Townsend MK, Curhan GC, Resnick NM, Grodstein F (2009) Type 2 diabetes mellitus and risk of stress, urge and mixed urinary incontinence. J Urol 181:193-197

2. Izci Y, Topsever P, Filiz TM, Çinar ND, Uludağ C, Lagro-Janssen $\mathrm{T}$ (2009) The association between diabetes mellitus and urinary incontinence in adult women. Int Urogynecol J Pelvic Floor Dysfunct 20:947-952

3. Fayyad AM, Hill SR, Jones GR (2009) Prevalence and risk factors for bothersome lower urinary tract symptoms in women with diabetes mellitus from hospital-based diabetes clinic. Int Urogynecol J Pelvic Floor Dysfunct 20:1339-1344

4. Ebbesen MH, Hannestad YS, Midthjell K, Hunskaar S (2007) Diabetes and urinary incontinence - prevalence data from Norway. Acta Obstet Gynecol Scand 86:1256-1262

5. Kebapci N, Yenilmez A, Efe B, Entok E, Demirustu C (2007) Bladder dysfunction in type 2 diabetic patients. Neurourol Urodyn 26:814-819

6. Smith DB (2006) Urinary incontinence and diabetes: a review. J Wound Ostomy Continence Nurs 33:619-623

7. Fayyad AM, Hill SR, Jones G (2010) Urine production and bladder diary measurements in women with type 2 diabetes mellitus and their relation to lower urinary tract symptoms and voiding dysfunction. Neurourol Urodyn 29:354-358

8. Siracusano S, d'Aloia G, Lentini MG, Silvestre G (2002) Diabetic cystopathy. Diabetes Nutr Metab 15:41-44

9. Liu RT, Chung MS, Lee WC, Chang SW, Huang ST, Yang KD et al (2011) Prevalence of overactive bladder and associated risk factors in 1359 patients with type 2 diabetes. Urology 78:1040 1045. doi:10.1016/j.urology.2011.05.017

10. Rizk DEE, Padmanabhan RK, Tariq S, Shafiullah M, Ahmed I (2006) Ultra-structural morphological abnormalities of the urinary bladder in streptozotocin-induced diabetic female rats. Int Urogynecol J Pelvic Floor Dysfunct 17:143-154

11. Subak LL, Whitcomb E, Shen H, Saxton J, Vittinghoff E, Brown JS (2005) Weight loss: a novel and effective treatment for urinary incontinence. J Urol 174:190-195

12. Brown JS, Wing R, Barrett-Connor E, Nyberg LM, Kusek JW, Orchard TJ et al (2006) Lifestyle intervention is associated with lower prevalence of urinary incontinence: the Diabetes Prevention Program. Diabetes Care 29:385-390 